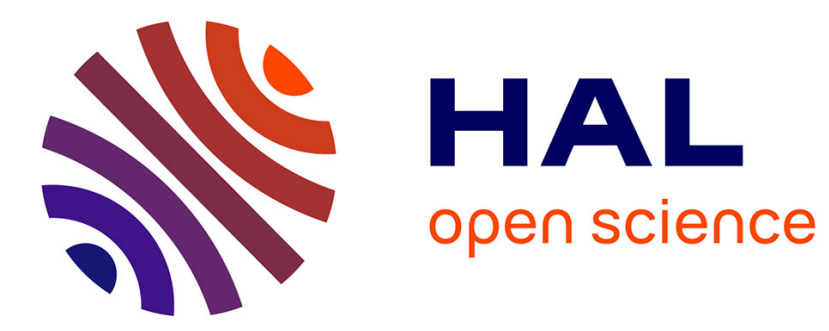

\title{
The influence of relational complexity and strategy selection on children's reasoning in the Latin Square Task
}

Patrick Perret, Christine Bailleux, Bruno Dauvier

\section{- To cite this version:}

Patrick Perret, Christine Bailleux, Bruno Dauvier. The influence of relational complexity and strategy selection on children's reasoning in the Latin Square Task. Cognitive Development, 2011, 26 (2), pp.127 - 141. 10.1016/j.cogdev.2010.12.003 . hal-01772235

\section{HAL Id: hal-01772235 \\ https://hal-amu.archives-ouvertes.fr/hal-01772235}

Submitted on 26 Apr 2018

HAL is a multi-disciplinary open access archive for the deposit and dissemination of scientific research documents, whether they are published or not. The documents may come from teaching and research institutions in France or abroad, or from public or private research centers.
L'archive ouverte pluridisciplinaire HAL, est destinée au dépôt et à la diffusion de documents scientifiques de niveau recherche, publiés ou non, émanant des établissements d'enseignement et de recherche français ou étrangers, des laboratoires publics ou privés. 


\title{
The influence of relational complexity and strategy selection on children's reasoning in the Latin Square Task
}

\author{
Perret, Patrick \\ Bailleux, Christine \\ Dauvier, Bruno
}

\section{Aix-Marseille Université}

\section{Introduction}

\subsection{Relational complexity and the development of reasoning}

The present study explored the influence of relational complexity on children's deductive reasoning. Recent perspectives on adult cognitive psychology (Oberauer, Süb, Wilhelm, \& Wittmann, 2008) suggest that the relational integration component of working memory constitutes a central mediator of human reasoning. In developmental research, this view also lies at the heart of relational complexity theory (RC theory; Halford, Wilson, \& Phillips, 1998), which states that growth in relational processing capacity with age increases the complexity of the mental models that children can form. In turn, these changes in the complexity of mental models (from which inferences are derived) increase children's understanding of the world, through conceptual refinements and the development of reasoning. In line with other contemporary views of reasoning (Johnson-Laird, 1983), RC theory is thus based on the assumption that, when solving problems, the human mind constructs mental models intended to represent the structure of the relations involved in these problems. A second core assumption is that limited processing capacity in working memory limits the complexity of these models. The relational complexity metric applies both to the 
structural properties of a problem and to individual processing capacity. Relational complexity is defined as the number of variables (or arguments) that must be related within the same cognitive representation. Unary relations are based on a single variable, binary relations on two variables, ternary relations on three, and so on.

Halford, Baker, McCreden, and Bain (2005) have established that quaternary relations are the most complex relations that adults can mentally represent, and constitute the upper limit of human processing capacity. However, RC theory has identified two mechanisms that can help individuals sidestep this processing barrier: segmentation and chunking.

Segmentation consists in breaking excessively complex tasks down into several steps, so as to reduce the relational complexity involved at each stage. Chunking consists in compressing two or more variables into one. This mechanism both reduces the processing load and allows the newly compressed variables to form a single argument in a higher-order relation. RC theory regards segmentation and chunking as important components of expertise in a particular conceptual domain or a specific type of task.

According to RC theory, age-related changes in processing capacity are a crucial factor for cognitive development, but not the only one. Halford has repeatedly stressed that $\mathrm{RC}$ theory does not deny the role played by knowledge or experience: processing capacity should be regarded as an enabling factor that gradually broadens the horizons of conceptual development and reasoning (e.g., Halford, 1999). Developmental changes in processing capacity are thought to occur approximately as follows: children become able to process unary relations at a median age of one year, binary relations at two years, ternary relations at five years, and quaternary relations at 11 years (Andrews \& Halford, 2002). This gradual growth in processing capacity enhances the number of variables that children can relate in their mental models, thereby allowing them to represent the structure of increasingly complex problems. As a consequence, their inferences in reasoning can rely on more accurate and adequate representations of the relational systems they are dealing with. 
$\mathrm{RC}$ theory thus provides a clear framework for predicting children's performance on reasoning tasks: performance should be determined mainly by the match (or mismatch) between the child's processing capacity and the task's relational complexity. Halford and Andrews (2004) revisited some of the most famous developmental tasks (e.g., class inclusion, transitive inference, theory of mind, etc.) and showed that accurate analysis of relational complexity can help to explain both early success and late failures on variants of these tasks. More recently, Birney, Halford, and Andrews (2006) developed a new experimental task, the Latin Square Task (LST), explicitly derived from RC theory and designed to test its predictions about the influence of relational complexity on deductive reasoning.

\subsection{The Latin Square Task}

This task is based on a matrix of 16 cells $(4 \times 4$ structure) that can be filled with four different geometric shapes. The defining principle is that each shape should appear only once in each row or column. Participants are confronted with an incomplete matrix and asked to determine which of the shapes should be placed in a target cell. The items are designed so that the information already present in the relevant rows or columns of the array can direct the participants' inferences towards the right conclusion. In its defining principle, the task resembles Sudoku problems and, as such, constitutes a "puzzle of pure deduction" (Lee, Goodwin, \& Johnson-Laird, 2008).

The LST has several important qualities with regard to RC theory requirements. First, the deductive mechanisms activated in this task are largely free from the influence of prior conceptual knowledge, pragmatic schemas or innate modules known to affect human reasoning and often difficult to disentangle in performance analyses. Second, the task also minimizes the amount of information to be held in memory and consequently maximizes the role of the processing (as opposed to storage) component of working memory in the determination of performance. Third, it relies on a single, simple rule (suitable for a broad 
range of ages and abilities) that can be applied to items of varying complexity. The relational complexity of LST items is manipulated by controlling the number of rows and columns that need to be simultaneously considered in order to choose the right shape for the target cell. "Binary items require integration of elements within either a single column or row [...]. Ternary items require integration of information from both a row and column [...]. For the quaternary items, solution is achieved by integrating elements across multiple rows and columns that are not necessarily fully constrained by a simple intersection" (Birney et al., 2006, p. 150-151).

Birney et al. (2006) studied the influence of relational complexity on the reasoning performance of university students and school children ranging in age from nine to 16 years old. Participants completed a total of 18 items, with six items per RC category (binary, ternary or quaternary). The results of this study, based on regression and Rasch analyses, confirmed the predictions of RC theory, insofar as items of greater complexity were associated with more errors and longer response times. However, the authors highlighted two instructive methodological difficulties. First, on the basis of empirical data, some of the items called for a reclassification (e.g., from quaternary to ternary), as participants could follow a valid but unanticipated reasoning pathway to find the solution. Using this alternative route of serial inferences meant that they encountered lower levels of relational complexity than the authors had envisaged when they designed the items. This phenomenon draws our attention to a crucial aspect of RC analysis, in that it applies to cognitive processes rather than to the task itself. As long as the items offer several possible paths to the solution, accurately estimating the level of relational complexity participants are really dealing with will remain an uncertain enterprise. Second, despite a clear complexity effect, the results indicated that factors other than dimensionality significantly contributed to the variations in performance on the LST. Response times were affected not just by RC but also by the number of empty cells in the matrix. Furthermore, the number of processing steps required to reach the solution 
significantly influenced both error rates and response times. For some items, intermediate empty cells had to be dealt with before the final inference concerning the target cell could be generated. Other items were single-step problems. Although this serial parameter was not explicitly controlled for in the generation of items in Birney et al.'s (2006) study, appropriate statistical analyses revealed that the participants' ability to undertake multistep reasoning made an important contribution to performance, above and beyond their processing capacity. A recent study by Zhang, Xin, Lin, and Li (2009) confirmed that the number of processing steps required to find a solution in the LST accounts for a major proportion of variability in item difficulty.

On the basis of Birney et al.'s (2006) results, we designed a new version of the LST in order to control for these nonrelational factors and to further explore the influence of complexity on children's reasoning. Experiment 1 describes the development of this new version of the task and reports the subsequent performances of a sample of school-aged children.

\section{Experiment 1}

The aim of Experiment 1 was to study the effect of relational complexity on children's performance after controlling for the effects of the nonrelational factors identified in Birney et al.'s (2006) study. To this end, three main changes were made to the original task:

(1) The number of empty cells was kept constant in all the items;

(2) All the items required a single inferential step to identify the right shape for the target cell (no intermediate cells to deal with);

(3) In order to constrain reasoning pathways (and consequently limit individual variability in the inferential routes chosen by the participants), only the relevant rows and columns of the matrix were shown. 
RC manipulations adhered to the principles defined by Birney et al. (2006), in that item complexity was a function of the number of rows and columns that had to be processed in parallel in order to perform the inferential step leading to the solution. We designed binary, ternary, and quaternary items (see Fig. 1 for examples of each category). In the ternary items, information from both a row and a column has to be integrated. We made an additional distinction between these ternary items: secant versus nonsecant, depending on the target cell's position with regard to the pieces of information that had to be integrated. As shown in Figure 1, secant ternary items were designed so that the target cell was located at the intersection of the row and column that had to be taken into account. In nonsecant ternary items, this was not the case. A preliminary study (Perret, Bailleux, \& Dauvier, 2008) had suggested that the position of the target cell in ternary items constitutes a neglected dimension of these items' difficulty. We therefore manipulated this second additional factor for ternary items to create a total of four categories.

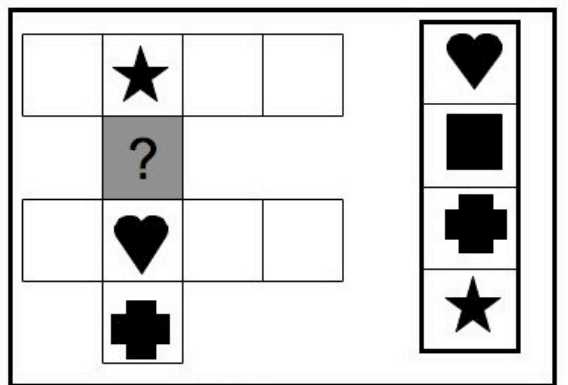

(a) binary

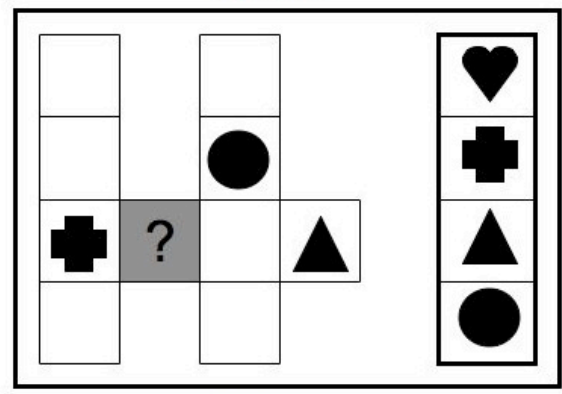

(c) Ternary - non secant

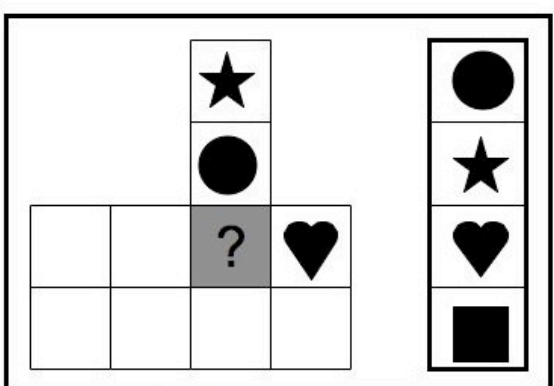

(b) Ternary- secant

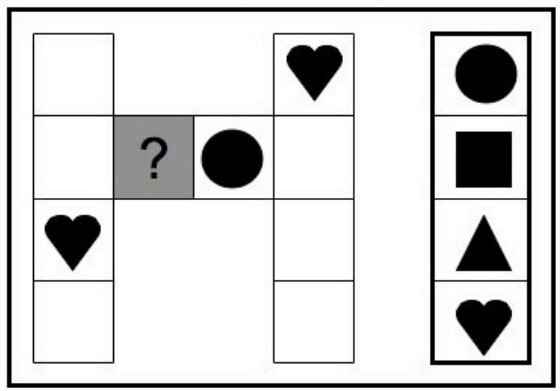

(d) quaternary

Figure 1: Examples of the categories of items used in Experiment 1. 


\subsection{Method}

\subsubsection{Participants}

Seventy-one French children aged between eight and 11 years took part in this experiment. The sample comprised 36 boys (51\%) and 35 girls (49\%). Children's average age was nine years and four months $(S D=15$ months $)$.

\subsubsection{Item generation}

A set of 24 items (six binary, six secant ternary, six nonsecant ternary, and six quaternary) was generated, following the basic principles set out in the previous section. We used six geometric shapes (square, circle, cross, triangle, star and heart), all of them blue in color. The order of item presentation was randomized, with the one proviso that none of the four conditions could occur more than three times in succession. We controlled the number of times that each shape appeared in the matrix structure and in the list of response options. The position of the filled cells was also controlled. All the items were built on a 4 x 4 structure, but only three rows and columns were displayed to participants, in order to reduce the number of noninformative cells and possible inferential pathways. We fixed the number of filled cells at three. Consequently, the number of empty cells was kept constant. The target cell was highlighted and indicated by a question mark in the center.

\subsubsection{Procedure}

E-Prime software (Psychology Software Tools, Inc., 2008) was used to build our version of the LST and the test was administered by a computer. The experiment began with a familiarization phase featuring four sample items (binary, secant ternary, nonsecant ternary and quaternary). Participants were encouraged to do their best and to respond as accurately as possible. The experimental design remained the same throughout and the instructions were relatively straightforward: "You have to find the shape missing from the cell with a question mark, obeying the following rule: each shape must appear only once in each row and in each column. You have to choose the missing shape from these four possibilities [participants were 
shown a list of four response options]. Be careful, there is only one right answer." The items were displayed on the left-hand side of the computer screen and the list of response options on the right-hand side. Participants responded by clicking on the relevant shape in the response options. All the participants completed the 24 items without any feedback from the experimenter.

\subsection{Results}

Several statistical approaches can be used to analyze successes and failures observed in a task like the LST. A simple experimental approach would be to compare the mean difficulties of groups of items in relation to their theoretical level of complexity. A gradual increase in observed difficulties as a function of relational complexity could be taken as evidence validating the theoretical analysis of the task. However, averaging across participants and items can hide differences between items and does not provide any information about the structure of individual differences. A more fine-grained approach consists in using psychometric tools, such as the Rasch model, a type of item response theory (IRT) model. In the Rasch model, each item is defined by its own level of difficulty, making it possible to pinpoint items that deviate from the prediction. Individual differences are also taken into account, as individual abilities are assumed to be continuously distributed across a latent continuum.

We therefore adopted a twofold statistical approach. First, we used repeated-measures ANOVAs to compare groups of items in a classic manner. Second, generalized linear mixedeffects models (GLMMs; Breslow \& Clayton, 1993), which can be regarded as a generalization of the multilevel model (Faraway, 2005) and some IRT models such as the

Rasch model (Boeck \& Wilson, 2004, p. 6; Miyazaki, 2005), were fitted to the data in order to validate the item classification. In the first analysis, individual mean proportions of correct responses were computed for the binary, ternary and quaternary items. These individual 
values were then averaged to obtain mean accuracy per complexity level (Fig. 2). Results clearly followed the expected stepwise increase in difficulty from binary to quaternary items. A repeated-measures ANOVA revealed a relatively strong and significant effect, $F(2,136)=$ $176, p<.001, \eta^{2}=.55$

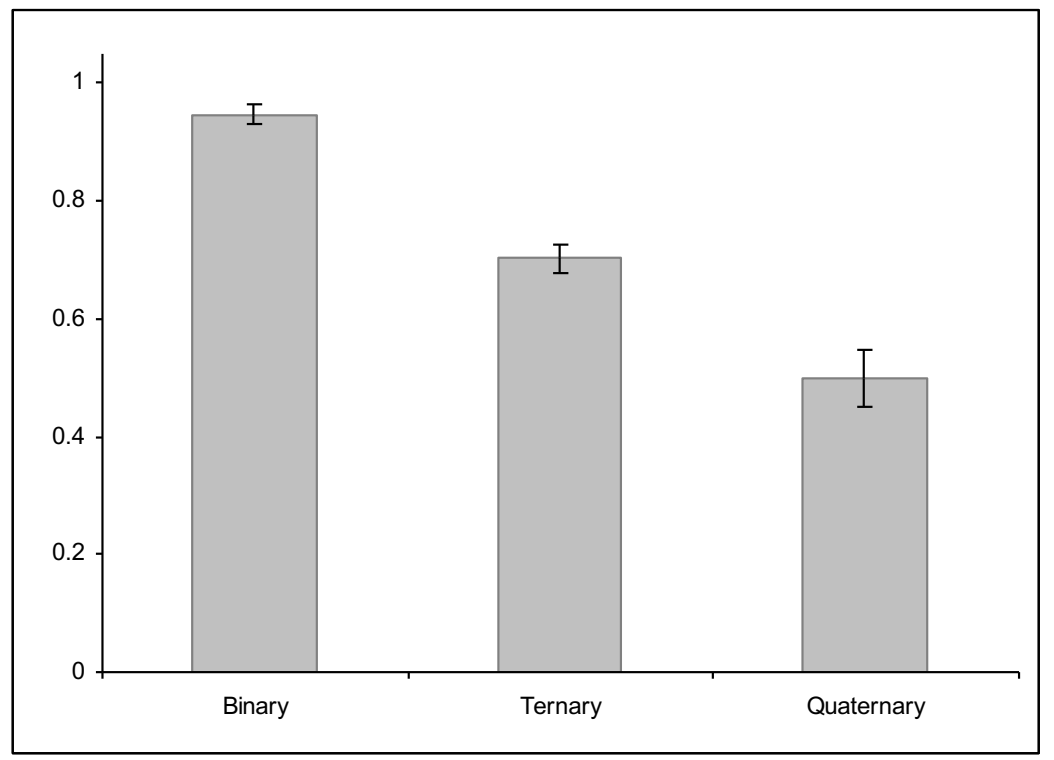

Note: The proportions of correct responses were first computed for each individual participant. This bar chart shows group mean accuracy with standard error for each level of complexity.

Figure 2: Mean (standard error) accuracy for each complexity level in Experiment 1.

An item-by-item examination of the data revealed that the group of ternary items encompassed very different levels of difficulty. It was immediately obvious that the distinction between secant and nonsecant items within the group of ternary items would have to be taken into account, as shown in Figure 3, so from then on, we distinguished between four types of item: binary (a), secant ternary (b), nonsecant ternary (c), and quaternary (d).

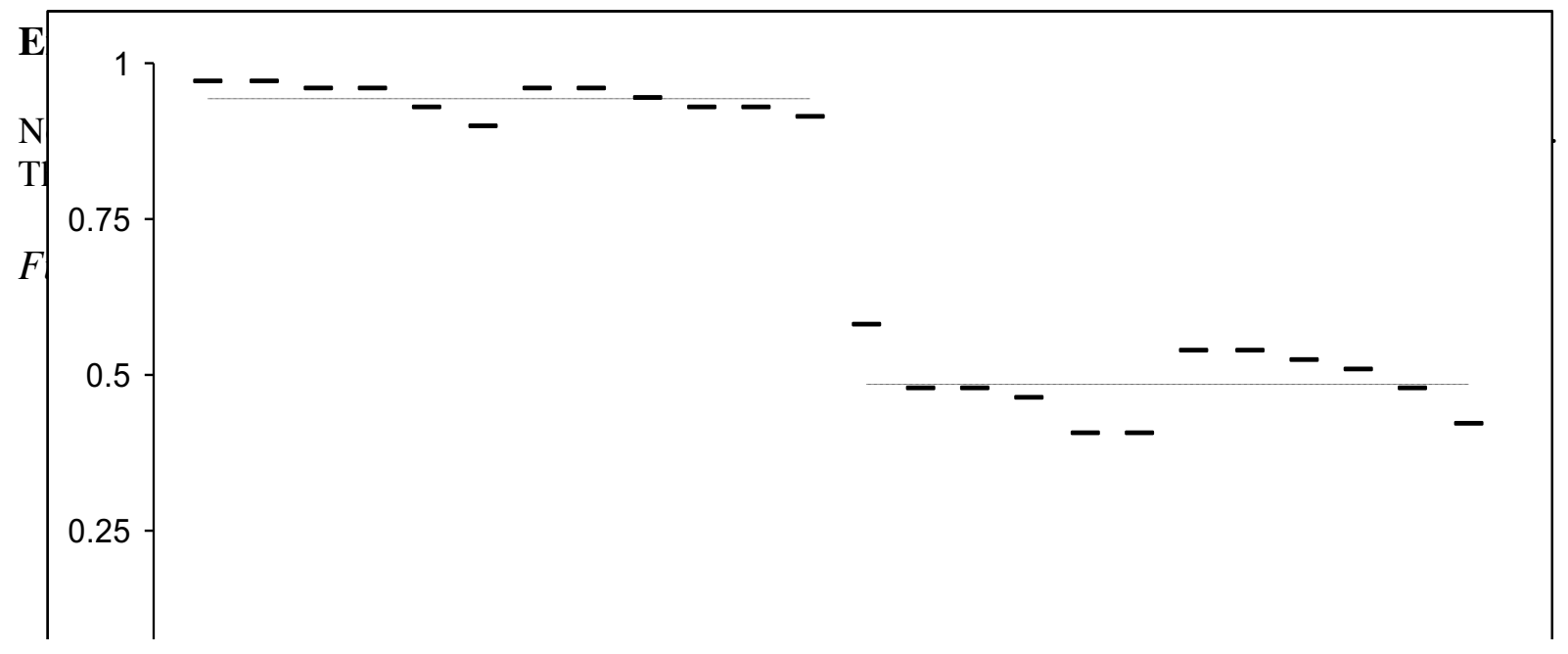


In order to empirically find the best form of item classification, three GLMMs (binomial distribution and logit link function) were fitted to the data, using the lme4 package I(Bates \& Sarkar, 2009) in R I(R Development Core Team, 2009). These models are very similar to IRT models such as the Rasch model, where item difficulty is taken into account by the fixedeffects parameters and participants' abilities by the random-effects parameters I(Boeck \& Wilson, 2004; Doran, Bates, Bliese, \& Dowling, 2007). Like IRT models, the GLMMs were directly fitted to the binary success/failure data without any averaging. This methodology offered some useful features in this context, allowing us to form groups of items and to force the difficulty parameters to the same level within a given group of items. In each of the three models, a different classification of items was used. A comparison of the models told us which classification was the most relevant, given the data.

In the first model (M1), items were classified according to their theoretical level of complexity. In the second model (M2), we added the distinction between secant ternary (b) and nonsecant ternary (c) items. In the last model (M3), binary (a) and secant ternary (b) items were set to the same level of difficulty, as were nonsecant ternary (c) and quaternary (d) items. A model comparison based on AIC and BIC criteria (Table 1) showed that M3 was the best model $(\mathrm{AIC}=1295, \mathrm{BIC}=1311) . \mathrm{M} 1$, which did not distinguish between ternary items, showed the poorest fit $(\mathrm{AIC}=1595, \mathrm{BIC}=1617)$ and was unsuitable for revealing individual differences (low between-participants variance: 1.5$)$. As M2 (AIC=1297, BIC=1324) was no better than M3, there seemed to be no empirical reason why we should make a distinction between binary and secant ternary on the one hand and nonsecant ternary and quaternary on the other. The mean observed proportion of correct responses for nonsecant ternary and quaternary items (.48) was higher than it would have been if children had used a guessing strategy (.25; there were four response options).

Table 1: Goodness of fit of the generalized linear mixed-effects models in Experiment 1. 


\begin{tabular}{cccccc}
\hline \multicolumn{6}{c}{ Generalized linear mixed-effect models } \\
\hline Model & Item classification & $d f$ & AIC & BIC & Participant variance \\
\hline M1 & (a) (b-c) (d) & 4 & 1595 & 1617 & 1.50 \\
M2 & (a) (b) (c) (d) & 5 & 1297 & 1324 & 2.41 \\
M3 & (a-b) $($ c-d) & 3 & 1295 & 1311 & 2.40 \\
\hline
\end{tabular}

\subsection{Discussion}

The items in our version of the Latin Square Task were created so as to minimize the impact of three factors known to have a confounding effect: the number of empty cells, the number of processing steps, and individual variability in inferential pathways. Results indicated that, when only the relational dimension of the items was considered, the data clearly confirmed the predictions of RC theory, as the mean proportion of correct responses decreased as a function of relational complexity. However, when the secant/nonsecant distinction between ternary items was taken into account, as suggested by the GLMMs, a more complex picture emerged: Binary and secant ternary items were found to be of equivalent difficulty for children, and a comparable equivalence emerged for nonsecant ternary and quaternary items. The latter results seem to argue against the moderating role of $\mathrm{RC}$ on performance.

One possible explanation is that the strategic dimension of the LST may have been neglected in our previous analyses of the task. On the basis of their work on Sudoku problems, Lee, Goodwin, and Johnson-Laird (2008) suggested that (i) individuals may recruit various strategies to cope with Latin square problems and (ii) variations in performances may be rooted in strategic shifts. With regard to the present task, we hypothesized that two types of strategies may have underlain children's reasoning: "cell-based reasoning " and "shape-based reasoning". The distinction between the two resides mainly in the initial focus of the child's attention: a given cell versus a given shape. Cell-based reasoning consists in (i) focusing attention on a given row or column in the array that will constitute the starting point for 
subsequent inferences, (ii) concentrating on a particular cell in this row or column, (iii) determining the shapes it could be filled with, given those already present in the same row or column, then, (iv) if more than one possibility is found, eliminating potential shapes by taking additional constraints from intersecting rows or columns into account, until only one shape is left. In the example below (Figure 4), taken from Experiment 1, the secant ternary item can be solved by (i) focusing attention on the first row of the matrix, (ii) concentrating on the target cell with a question mark, (iii) determining the shapes it could be filled with, given those already present in the row (either a circle or a heart), then (iv) eliminating the circle by virtue of its occurrence in the intersecting column, so that the square is the only possibility left.
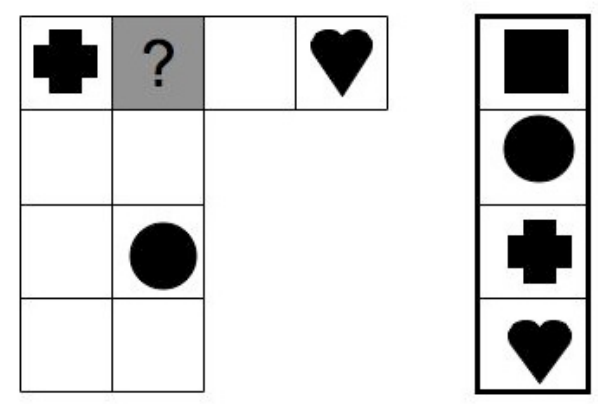

Figure 4. Example of a secant ternary item in Experiment 1.

Shape-based reasoning consists in (i) focusing attention on a given row or column in the array that will constitute the starting point for subsequent inferences, (ii) concentrating on a particular shape among the response options, (iii) determining the cells where it could be placed, given those already filled in the row or column in question, then, (iv) if there is more than one possibility, eliminating potential cells by taking into account additional constraints from intersecting rows or columns, until there is only one cell left. The quaternary item (Fig. 5), for example, can be solved by (i) focusing attention on the one row in the matrix, (ii) concentrating on the heart option, (iii) identifying the three cells where the heart could be placed, given the presence of the circle, and (iv) eliminating the two end cells by virtue of the 
hearts that are already present in the intersecting columns, so that the only possibility for the heart in the row in question is the cell with the question mark.
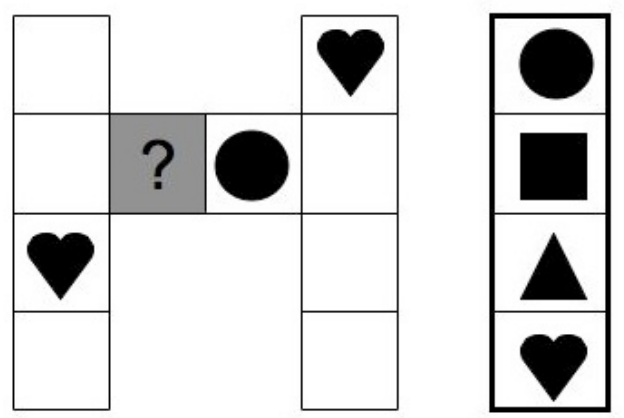

Figure 5. Example of a quaternary item in Experiment 1.

The essence of the distinction described here can be summed up as follows: looking for possible shapes for a given cell versus looking for possible cells for a given shape. We contend that this strategic distinction may help to explain the data from Experiment 1. Whereas binary and secant ternary items can be solved using cell-based strategies, nonsecant ternary and quaternary items require shape-based strategies, as can be seen in the quaternary item above: once the child has identified three possible candidates to fill the target cell (the heart, the star, and the square), no additional constraint is available to disambiguate the decision. Thus, these categories of items require a shape-based reasoning strategy. Furthermore, the shape-based strategy may allow children to chunk the information available from multiple rows and columns when producing the final inference: the shape under consideration can go nowhere else except in the target cell. It should be noted that when the inference is made, the number of intersecting rows and columns that have previously been considered does not constrain the reasoning because this information has been reduced to a single argument ("the shape can go nowhere else except..."). This chunking mechanism could explain why complexity effects were not clearly observed in Experiment 1. As Halford and 
Andrews (2004) point out, "it has been a major difficulty for cognitive complexity analyses that humans have very proficient strategies to reduce processing loads" (p. 129). In Experiment 2, we tested the hypothesis that performance on the LST may rely on deductive strategies that reduce the processing demands of complex items.

\section{Experiment 2}

Experiment 2 was designed to examine whether school-aged children of a comparable age range were able to solve quinary items. We have suggested that the dilution of complexity effects in Experiment 1 may have resulted from a reduction in the processing demands of excessively complex items. If this interpretation is correct, then increasing item complexity beyond the previous four dimensions would not preclude success, even though this level of complexity clearly surpasses children's processing capacity, according to RC theory. A further aim of Experiment 2 was to replicate the results of Experiment 1, using a visual display of the task which more closely resembled that used by Birney et al. (2006). In ad hoc interviews, some children in Experiment 1 indicated that, as they were performing the task, they developed a heuristic that consisted in looking through the response options and choosing the shape that was missing from the array. Roberts (2000) emphasized the fact that many deduction tasks give rise to the development of such shortcut perceptual strategies in order to "obtain a solution directly from the problem statements" (p. 25). Due to our simplified display of the task, a systematic use of this heuristic (i.e., "choosing the shape that isn't already in the array") would have yielded accurate responses for binary and secant ternary items, though not for nonsecant ternary and quaternary ones (where the correct responses corresponded to shapes that were already present in the array). In Experiment 2, we wanted to rule out the possibility that our results were the byproduct of a simplified display. Thus, we used a complete matrix (with five rows and columns) and increased the number of filled cells so as 
to avoid the development of such shortcut strategies. Nevertheless, drawing on Birney et al.'s (2006) results, we retained the objective of limiting the impact of extraneous factors that were likely to have a confounding effect. To this end, the LST items in Experiment 2 had the following features:

(1) The size of the matrix was increased ( $5 \times 5$ structure) to allow the creation of quinary items (i.e., requiring participants to consider the possible elements in the target row or column whilst also taking elements in four other rows or columns into consideration);

(2) The number of cells provided per item was increased, but held constant for all the items;

(3) Contrary to Experiment 1, a complete matrix of rows and columns was presented, but in order to limit individual variability in the inferential pathways chosen by the participants, we highlighted the relevant row or column that had to be considered first;

(4) All the items required a single inferential step to identify the right shape for the target cell (i.e., no intermediate cell to deal with);

(5) Relational complexity was manipulated according to the principles defined by Birney et al. (2006), and four categories of items were used (with six items per category): secant ternary, nonsecant ternary, quaternary and quinary (see Fig. 5 for an example of each category). Binary items were excluded from this experiment, as a clear floor effect had emerged in Experiment 1. 


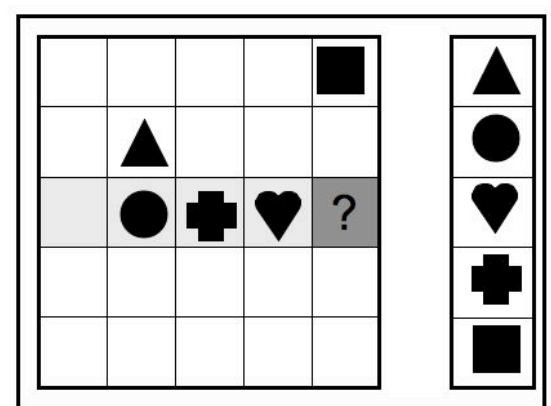

(a) Ternary- secant

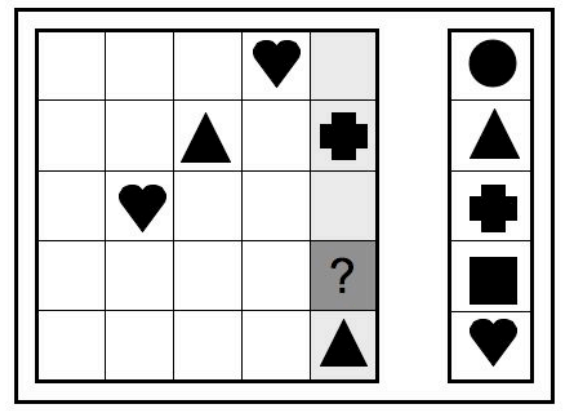

(c) Quaternary

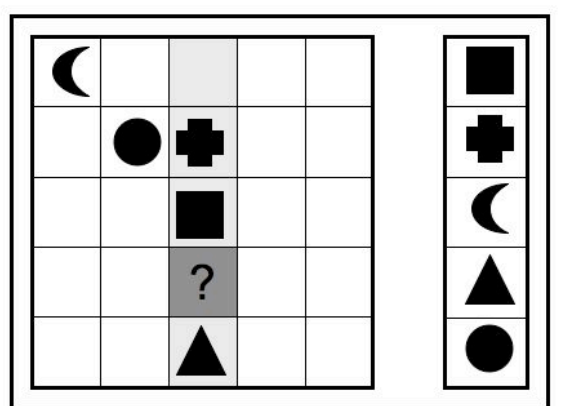

(b) Ternary- nonsecant

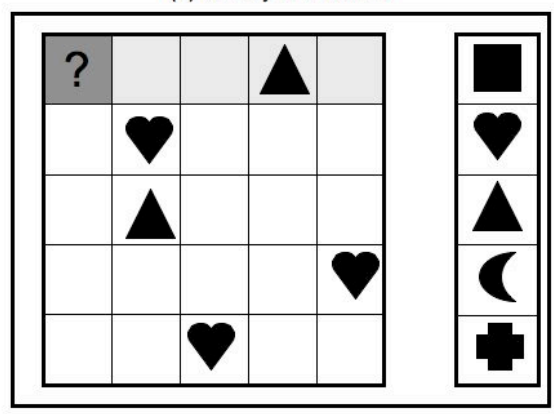

(d) Quinary

Figure 6. Examples of the four categories of items used in Experiment 2.

\subsection{Method}

\subsubsection{Participants}

Eighty-nine French children aged between eight and 12 years took part in this experiment. The sample comprised 40 boys (45\%) and 49 girls (55\%). Their average age was 10 years and one month $(S D=12$ months $)$.

\subsubsection{Item generation}

A set of 24 items (six secant ternary, six nonsecant ternary, six quaternary, and six quinary) was generated in the same way as in the first experiment. In this version, we used six blue shapes (square, circle, cross, triangle, heart, and moon) and added an additional control: in the ternary items, the response was always one of the shapes already present in the array (in order to preclude the use of the heuristic described in the Discussion of Experiment 1). All items were based on a 5 x 5 structure. All the cells were visible, but we highlighted the most informative row or column in a pale yellow color, in order to guide the participants' reasoning and reduce variability in inferential pathways. We fixed the number of filled cells at five, with 
the result that the number of empty cells was also constant. The target cell was indicated by a question mark and highlighted in a luminous yellow.

\subsubsection{Procedure}

The procedure was the same as in Experiment 1, with a familiarization phase featuring six practice items (two binary, one secant ternary, one nonsecant ternary, one quaternary and one quinary). All the participants then completed the 24 items, without any feedback from the experimenter.

\subsection{Results}

At the group level, no difference in the level of difficulty was observed between the nonsecant ternary, quaternary and quinary items. Mean accuracies were very similar, at around .5. A repeated-measures ANOVA revealed a significant effect of complexity level, $F(3,264)=35.8, p<.001, \eta^{2}=.17$, due to the high success rate observed for the secant ternary items (.84).

To ensure similar levels of difficulty within each group of items and to validate item classification, three GLMMs were fitted to the data. As shown in Figure 7, secant ternary items appeared to have a homogeneous level of difficulty. Nonsecant ternary, quaternary and quinary items, however, showed greater within-group variability across items. In the first model (M1), items were categorized in the four previously mentioned categories: secant ternary (b), nonsecant ternary (c), quaternary (d), and quinary (e). In the second model (M2), nonsecant ternary (c), quaternary (d) and quinary (e) items were grouped into a single class and their difficulty levels were constrained to equality. This classification was strongly suggested by the descriptive statistics. The third model (M3) was a Rasch model, where each item had its own difficulty parameter. The Rasch model allowed us to test within-group variability across items. If this variability was too high, it would not be appropriate to subject the difficulty levels to a single-value constraint within a given group of items, and a more flexible model like the Rasch model would show a better fit. 


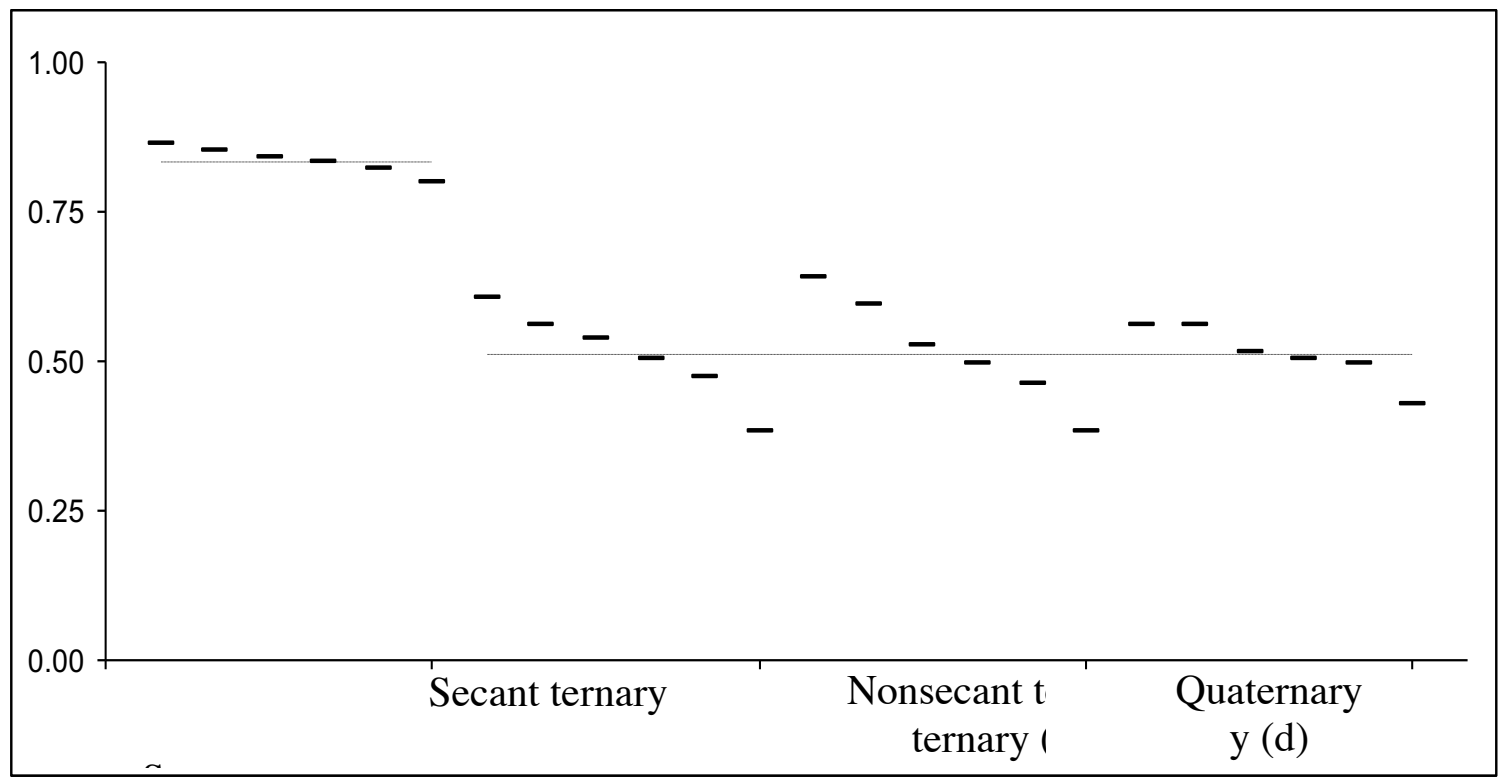

Figure 7: Mean accuracy per item in Experiment 2.

The best model according to both AIC and BIC criteria was M2 (Table 2), which meant that no distinction in mean difficulty levels needed to be made between nonsecant ternary, quaternary and quinary items at the group level. The Rasch model (M3; AIC $=2439$, $\mathrm{BIC}=2581)$ was no better than $\mathrm{M} 2(\mathrm{AIC}=2438, \mathrm{BIC}=2455)$. The apparent heterogeneity shown in Figure 7 within the nonsecant ternary, quaternary and quinary groups of items was not great enough to require a more fine-grained classification. This apparent heterogeneity was probably due to the fact that the difficulty levels of these items were distributed around .5 . Variability is necessarily greater at the center of the scale than it is at the two ends.

Table 2: Goodness of fit of the generalized linear mixed-effects models and finite mixture of generalized linear models in Experiment 2.

\begin{tabular}{cccccc}
\hline \multicolumn{5}{c}{ Generalized linear mixed-effects models } \\
\hline Model & Item classification & $d f$ & AIC & BIC & Participant variance \\
\hline M1 & (b) (c) (d) (e) & 5 & 2442 & 2470 & 1.24 \\
M2 & (b) (c-d-e) & 3 & 2438 & 2455 & 1.24 \\
M3 (Rasch) & item by item (24) & 25 & 2439 & 2581 & 1.31 \\
\hline \multicolumn{5}{c}{ Finite mixtures of generalized linear models } \\
\hline
\end{tabular}




\begin{tabular}{cccccc}
\hline Model & Item classification & $d f$ & AIC & BIC & Number of classes \\
\hline M4 & (b) (c) (d) (e) & 9 & 2373 & 2424 & 2 \\
M5 & (b) (c) (d) (e) & 14 & 2335 & 2414 & 3 \\
M6 & (b) (c) (d) (e) & 19 & 2298 & 2406 & 4 \\
M7 & (b) (c) (d) (e) & 24 & 2290 & 2426 & 5 \\
\hline
\end{tabular}

In order to test our hypothesis on individual strategic differences, two additional analyses were conducted: regression analyses and finite mixtures of generalized linear regression models. Whereas the secant ternary items could be solved using a cell-based reasoning strategy, we hypothesized that the nonsecant ternary items would require a shapebased strategy comparable to the one required by the quaternary and quinary items. As a consequence, individual performances on nonsecant ternary items would be a better predictor of the performances on quaternary and quinary ones, which hypothetically involve the same inferential processes. ${ }^{1}$ We then conduced two regression analyses, with secant and nonsecant ternary scores as predictors. The score on nonsecant ternary items significantly predicted performances on quaternary and quinary items. Standardized regression coefficients were .33 $(p=.001)$ and $.25(p=.015)$, respectively. Conversely, the score on secant ternary items was not predictive, as standardized regression coefficients were not significant: .11 and $.10(p>$ .05 for both).

The GLMMs previously fitted to the data were not necessarily appropriate for examining individual differences in strategy use. These models assume that individual differences are ordered along a latent continuum. However, if the availability of the shape-based strategy differs from one child to another, individual differences have to be viewed as qualitative differences between groups of children. We tested the hypothesis of discrete ability levels, using a type of latent class model called "finite mixtures of generalized linear regression models" I(FM-GLM; Grün \& Leisch, 2008). This methodology provided a means of

\footnotetext{
${ }^{1}$ We thank Graeme Halford for this suggestion.
} 
comparing models with different numbers of classes of children and with specific levels of item difficulty within each class. We implemented the FM-GLMs using the FlexMix R package I(Grün \& Leisch, 2007; Leisch, 2004). Another difference between FM-GLMs and GLMMs is that in the former, the levels of item difficulty are computed within each class. Hence, there is no more absolute item characteristic. This means that two items can be ordered differently in two different classes regarding their level of difficulty. This was a useful feature, given our theoretical assumption that the perceived difficulty of, say, a quaternary item might be very different for a child using the shape-based strategy as opposed to a child whose reasoning was based solely on the cell-based strategy. Although the number of parameters increases rapidly with the number of classes, the goodness-of-fit criteria used to select the model (i.e., AIC and BIC) take parsimony into account.

Four models were fitted to the data, with between two and five classes. The four FMGLMs all had better fits than M2, suggesting that a discrete representation of individual differences was more appropriate. According to the AIC criterion, the five-class model (M7; AIC $=2290)$ was slightly better than the four-class one (M6; AIC = 2298), while the latter exhibited the smallest BIC score (2406). The four-class model was thus selected as the best model, favoring parsimony and based therefore on the BIC criterion. Table 3 provides a description of these four groups of children.

Table 3: Description of the four groups revealed by latent class analyses in Experiment 2.

\begin{tabular}{lcccc}
\hline & Class 1 & Class 2 & Class 3 & Class 4 \\
\hline Number of children & 24 & 7 & 30 & 28 \\
Age (in months): mean & 123.96 & 118.14 & 119.47 & 120.57 \\
Age (in months): standard deviation & 9.80 & 5.40 & 13.04 & 12.87 \\
\hline Accuracy on secant ternary & .86 & .24 & .87 & .93 \\
Accuracy on nonsecant ternary & .77 & .26 & .46 & .41 \\
Accuracy on quaternary & .92 & .26 & .12 & .67 \\
Accuracy on quinary & .91 & .31 & .13 & .63 \\
\hline
\end{tabular}

The first class (C1) contained 24 children, who achieved high accuracy levels on every item, even the quinary ones (accuracy ranging from .77 to .91). A smaller class of seven 
children (C2) was made up of participants who exhibited a low level of accuracy on all four item types. The accuracy levels we observed in this second class, ranging from .24 to .31 , were close to the expected chance score of .25 . The third class (C3) contained 30 children who correctly solved secant ternary items most of the time (accuracy of .87) but had far greater difficulty with nonsecant ternary items (.46) and with quaternary and quinary ones (.12 and .13). The last class (C4) was composed of 28 children who performed very well on secant ternary items (.93), but moderately so on nonsecant ternary items (.41), like the children in $\mathrm{C} 3$. However, the children in $\mathrm{C} 4$ differed from their $\mathrm{C} 3$ counterparts on quaternary and quinary items, as they managed to solve them most of the time (accuracies of .67 and $.63)$.

Mean age and standard deviation for each class are provided in Table 3. Descriptively, results showed the highest mean age (123.96 months) for C1, followed by C4 (120.57 months) and C3 (119.47 months) and finally C2, corresponding to children who performed at chance level (118.14 months). However, an analysis of variance indicated that these age differences between classes were not significant, $F(3,85)=.8, p<1$, ns.

\subsection{Discussion}

Although we modified the visual display of the task so that it precluded recourse to a shortcut heuristic, the results of Experiment 2 broadly replicated the data of our first experiment. A significant difference in children's performances once again emerged between the two categories of ternary items (secant vs. nonsecant), even though they shared similar levels of complexity. Furthermore, the model of item classification that best fitted the data made no distinction between nonsecant ternary, quaternary, and quinary items with regard to their levels of difficulty, despite their differences in relational complexity. This result, in conjunction with the relatively high success rate for quinary items, confirmed the hypothesis formulated in the Discussion of Experiment 1 that specific deductive mechanisms can free 
performance from the constraint of limited processing capacity. A central assumption of RC theory is that quaternary relations constitute the upper limit of human processing capacity. Beyond this level of complexity, segmentation or chunking strategies are needed to reduce the task's processing load. The fact that performance on nonsecant ternary items significantly predicted performance on quaternary and quinary ones further suggests that children's reasoning on those items relied on comparable mechanisms of complexity reduction. With reference to Birney et al.'s (2006) formalization principles, ${ }^{1}$ chunking mechanisms involved in the categories of items represented in Figure 6 could be expressed as follows (with chunks indicated by continuous underlining ${ }^{1}$ ):

- Secant ternary: not (circle, cross, heart, square) $\rightarrow$ triangle (cell-based reasoning);

- Nonsecant ternary: not (cross, square, triangle, moon in 1.3) $\rightarrow$ moon in 4.3 (shape-based reasoning);

- Quaternary: not (cross, triangle, heart in 1.5 or 3.5) $\rightarrow$ heart in 4.5 (shape-based reasoning);

- Quinary: not (triangle, heart in 1.2,1.3 or 1.5) $\rightarrow$ heart in 1.1 (shape-based reasoning).

It comes out from these renewed RC analyses that nonsecant ternary, quaternary, and quinary items (i) can be chunked down to equivalent levels of complexity (i.e., ternary), despite differences in dimensionality, and (ii) differ from secant ternary items by virtue of a qualitatively different inferential process (shape-based reasoning).

Another key aspect of the present data concerns the results of the latent class analyses. These revealed four different patterns of performance, clearly suggesting individual variability in the way children coped with our four categories of items. In line with the hypothesis formulated in the Discussion of Experiment 1, these performance profiles may reflect the differential availability of cell- and shape-based strategies to support children's

\footnotetext{
${ }^{2}$ We are grateful to Graeme Halford for his help in applying RC analyses to the inferential strategies we identified.
} 
inferences, with shape-based strategies representing a more sophisticated form of reasoning. One possible explanation is that shape-based reasoning involves an additional inferential step: if a shape cannot occur in any of the other empty cells in a row or column, it has to occur in the cell in question. Within this framework, the four groups of participants appear to have been characterized by contrasting levels of strategy mastery. Class 2 children were unable to apply either of the deductive strategies to this task. Class 3 children were able to use cellbased reasoning for secant ternary items, but failed to apply or switch to a shape-based strategy when confronted with nonsecant ternary, quaternary, or quinary items. By contrast, Class 1 children were able to switch efficiently between these two strategies according to item requirements. Children in Class 4 presented the most surprising pattern of performance, with a sort of U-shaped curve for performance as a function of relational complexity. They succeeded better on quaternary and quinary items than on nonsecant ternary ones. One interpretation of this pattern is that the quaternary and quinary items evoked a shape-based strategy more strongly, insofar as the shape that children needed to take into account appeared two or three times in the array, and may therefore have attracted their attention. A rather similar phenomenon was reported by Lee, Goodwin, and Johnson-Laird (2008) in their work on Sudoku problems. The authors noticed that, despite an overall complexity effect, an increase in relational complexity was not always associated with an increase in item difficulty. "The departure from the effects of relational complexity when its value was five is probably attributable to the ease of noticing four instances of the same digit in columns and rows intersecting the box containing the target cell" (p. 353).

Interestingly, the four groups of children identified by the latent class analyses did not significantly differ in age. Given the age range of our sample, developmental changes in processing capacity between 8 and 12 years should have induced age-related latent classes if relational complexity was the only factor influencing performance. Conversely, if specific inferential strategies allowed a reduction in the relational complexity that children needed to 
process, no such developmental trend would be expected. Our results suggest that there are no clear developmental constraints to preclude the emergence of either cell- or shape-based strategies, at least for children in the age range considered in our sample.

\section{General discussion}

Drawing on Birney et al.'s (2006) research, the present study further documented the influence of relational complexity on children's deductive reasoning in the LST. With regard to previous studies (Biney et al., 2006 ; Zhang, Xin, Lin, \& Hong, 2009), two main changes were introduced into the task. First, we controlled for the effects of nonrelational factors that were likely to have confounding effects. Second, we introduced a distinction between two categories of ternary items, based on the target cell's position with regard to the information that needed to be integrated: secant versus nonsecant ternary items. Compared with previous research, the results of both experiments revealed a dilution of complexity effects, insofar as (i) items of the same complexity were characterized by different levels of difficulty and (ii) items of different complexity were characterized by comparable levels of difficulty. Children's mean performances on quinary items in Experiment 2 (which were comparable to their performances on nonsecant ternary and quaternary items) confirmed that their inferences were based on reasoning mechanisms that reduced the task's theoretical processing load. Furthermore, latent class analyses revealed the existence of considerable individual variability in the response patterns for the various categories of items. Thus, any theoretical account of the present data needs to explain not only why one category of items was more difficult than another, but also why one particular pattern applied to some children but not to others. We propose that children's reasoning on the LST relied on two deductive strategies, focusing either on the possible shapes for a given cell or on the possible cells for a given shape. We argue that the latter strategy reduced the items' theoretical processing load because the final inferential step allowed previous information to be chunked into a single argument. This 
chunking mechanism could explain the dilution of complexity effects observed in both our experiments. The foregoing outline also provides a possible explanation for individual variability in response patterns, in that it could reflect the differential availability and/or flexibility of use of the cell- and shape-based reasoning strategies.

Two implications, one methodological the other theoretical, can be drawn from the present study. At the methodological level, Birney et al. (2006) regarded the LST as a promising psychometric tool for assessing the influence of the processing component of working memory. We share the view that future explorations of how the working memory processing dimension influences the development of reasoning would benefit from a task specifically designed to assess children's processing capacity. However, given the present data, it may be premature to recommend the use of the LST in this context, for unless chunking strategies are controlled for, children's performances on the task cannot be regarded as a valid measure of their processing capacity. This difficulty was clearly expressed by Halford, Baker, McCredden, and Bain (2005), in their investigation of the limits of human processing. "Assessment of processing capacity is difficult because of the great power of strategies for reducing processing load, thereby optimizing use of available capacity" (p. 70). Halford and his colleagues have repeatedly stressed that the relevance of RC analyses depends on the accuracy of the process models of the tasks that are independently constructed. From this perspective, the present study may contribute to our understanding of the cognitive processes that are actually used by children in the LST, and provide useful constraints for future analyses of complexity.

At a more theoretical level, Markovits and Barrouillet (2004) have called for a revival in the developmental study of reasoning and put forward three possible theoretical frameworks within which to address the question of "what develops" in children's reasoning: (i) accounts that emphasize age-related changes in executive control, (ii) metalogical approaches which regard children's progress as the expression of an enhanced understanding of their own 
inferences, and (iii) neo-Piagetian perspectives, according to which age-related increases in attentional resources constitute a key factor. In the light of the present discussion, a fourth approach clearly needs to be mentioned: the adaptive selection of strategies from the child's repertoire (Siegler, 1996). Although these four different approaches to reasoning development have given rise to specific research programs in the past, they are in no way mutually exclusive in their accounts of the changes that affect children's reasoning. RC theory, for example, does not preclude knowledge effects but works in combination with them (Halford \& Andrews, 2004), as illustrated by the present study. Given the clear interaction between several factors influencing children's performances on the LST, we contend that this task could constitute a fruitful experimental context for studying their dynamic transactions. The present study highlighted how the strategic dimension of the task influences the relational complexity that children need to process and, as such, mediates processing load constraints. Future research could focus on how far children's adaptive shifting from one strategy to another as a function of the items' requirements (i.e., the executive dimension of the task) relies on an increased awareness of their own inferential processes (i.e., metalogical development). 


\section{References}

Andrews, G., \& Halford, G. S. (2002). A cognitive complexity metric applied to cognitive development. Cognitive Psychology, 45, 153-219.

Bates, D., \& Sarkar, D. (2009). lme4: Linear mixed-effects models using S4 classes. Retrieved from lhttp://CRAN.R-project.org/

Birney, D. P., Halford, G. S., \& Andrews, G. (2006). Measuring the influence of complexity on relational reasoning: The development of the Latin Square Task. Educational and Psychological Measurement, 66, 146-171.

Boeck, P. D., \& Wilson, M. (Eds.) (2004). Explanatory item response models: A generalized linear and nonlinear approach. New York: Springer.

Breslow, N. E., \& Clayton, D. G. (1993). Approximate inference in generalized linear mixed models. Journal of the American Statistical Association, 88(421), 9-25.

Doran, H., Bates, D., Bliese, P., \& Dowling, M. (2007). Estimating the multilevel Rasch model: With the lme4 package. Journal of Statistical Software, 20(2).

Faraway, J. J. (2005). Extending the linear model with R: Generalized linear, mixed effects and nonparametric regression models. New York: Chapman \& Hall/CRC.

Grün, B., \& Leisch, F. (2008). Finite mixtures of generalized linear regression models. In Shalabh, \& C. Heumann (Eds.), Recent advances in linear models and related areas (pp. 205-230). Berlin: Springer.

Grün, B., \& Leisch, F. (2007). Fitting finite mixtures of generalized linear regressions in $R$. Computational Statistics \& Data Analysis, 51(11), 5247-5252.

Halford, G. S. (1999). The development of intelligence includes the capacity to process relations of greater complexity. In M. Anderson (Ed.), The development of intelligence (pp. 193-213). Hove, UK: Psychology Press. 
Halford, G. S., \& Andrews, G. (2004). The development of deductive reasoning: How important is complexity? Thinking and Reasoning, 10, 123-145.

Halford, G. S., Baker, R., McCredden, J. E., \& Bain, J. D. (2005). How many variables can humans process? Psychological Science, 16, 70-76.

Halford, G. S., Wilson, W. H., \& Phillips, S. (1998). Processing capacity defined by relational complexity: Implications for comparative, developmental, and cognitive psychology. Behavioral and Brain Sciences, 21, 803-865.

Johnson-Laird, P. N. (1983). Mental models. Cambridge, UK: Cambridge University Press.

Lee, N. Y., Goodwin, G. P., \& Johnson-Laird, P. N. (2008). The psychological puzzle of Sudoku. Thinking \& Reasoning, 14, 342-364.

Leisch, F. (2004). \{FlexMix $\}$ : A general framework for finite mixture models and latent class regression in R. Journal of Statistical Software, 11(8), 1-18.

Markovits, H., \& Barrouillet, P. (2004). Introduction: Why is understanding the development of reasoning important? Thinking and Reasoning, 10, 113-121.

Miyazaki, Y. (2005). Some links between classical and modern test theory via the two-level hierarchical generalized linear model. Journal of Applied Measurement, 6(3), 289310.

Oberauer, K., Süb, H-M., Wilhelm, O., \& Wittmann, W. W. (2008). Which working memory functions predict intelligence? Intelligence, 36, 641-652.

Perret, P., Bailleux, C., \& Dauvier, B. (2008). The Latin Square Task and the measure of processing capacity in $R C$ theory. Paper presented at the 18th advanced course of the Archives Jean Piaget on Cognitive Development: Mechanisms and Constraints, Geneva, Switzerland.

R Development Core Team. (2009). R: A language and environment for statistical computing. Vienna, Austria: $\mathrm{R}$ Foundation for Statistical Computing. Retrieved from Ihttp://www.R-project.org 
Rasch, G. (1961). On general laws and the meaning of measurement in psychology. Proceedings of the Fourth Berkeley Symposium on Mathematical Statistics and Probability (pp. 321-334). Berkeley: University of Chicago Press.

Roberts, M. J. (2000). IIndividual differences in reasoning strategies: A problem to solve or an opportunity to seize? In W. Schaeken, G. De Vooght, A. Vandierendonck, and G. d'Ydewalle, (Eds.), Deductive reasoning and strategies (pp. 23-48). Mahwah, NJ: Lawrence Erlbaum.

Siegler, R. S. (1996). Emerging minds: The process of change in children's thinking. New York: Oxford University Press.

Zhang, L., Xin, Z., Lin, C., \& Li, H. (2009). The complexity of the Latin Square Task and its influence on children's performance. Chinese Science Bulletin, 54, 766-775 